\title{
UNION THEOREM FOR COHOMOLOGICAL DIMENSION: A SIMPLE COUNTEREXAMPLE
}

\author{
JERZY DYDAK
}

(Communicated by James E. West)

\begin{abstract}
An elementary counterexample to the Union Theorem for cohomological dimension with coefficients in $\mathrm{Z} / 2^{\infty}$ is presented.
\end{abstract}

Recently, Dranishnikov, Repovš, and Ščepin [DRS] have found a subset $A$ of $R^{4}$ such that $\operatorname{dim}_{\mathbf{Q} / \mathbf{Z}} A=\operatorname{dim}_{\mathbf{Q} / \mathbf{Z}}\left(R^{4}-A\right)=1$ which demonstrates that the Union Theorem $\left(\operatorname{dim}_{G}(A \cup B) \leq \operatorname{dim}_{G} A+\operatorname{dim}_{G} B+1\right)$ is not valid for $G=$ $\mathbf{Q} / \mathbf{Z}$. Previously, partial validity of the Union Theorem had been established by Rubin [Ru] $(G=\mathbf{Z})$ and Dydak and Walsh [DW] $(G=\mathbf{Z} / p, G$ a subring of $\mathbf{Q}$ ).

The reasoning in [DRS] is quite ingenious and uses recent results of the authors concerning embedding problems for cohomological dimension. The purpose of this note is to present a simple construction of a subset $A$ of $S^{4}$ such that $\operatorname{dim}_{\mathrm{Z} / 2} A=1$ and $\operatorname{dim}_{G}\left(S^{4}-A\right)=1$ for any abelian group $G$ divisible by 2 . Taking $G=\mathbf{Z} / 2^{\infty}$ (i.e., the direct limit of $\mathbf{Z} / 2 \rightarrow \mathbf{Z} / 4 \rightarrow \cdots \rightarrow \mathbf{Z} / 2^{k} \rightarrow$ ... ) one obtains a counterexample to the Union Theorem for cohomological dimension. Indeed, Bockstein Inequalities [Ku] imply that $\operatorname{dim}_{\mathrm{Z} / 2^{\infty}} A=1$.

Basic Lemma. Given a compact PL submanifold $M^{4}$ of $S^{4}$ there is a compactum $X \subset S^{4}-M$ such that $\operatorname{dim}_{\mathrm{Z} / 2} X=1$ and the inclusion induced homomorphism

$$
H^{1}\left(S^{4}-X ; G\right) \rightarrow H^{1}(M ; G)
$$

is an epimorphism for any abelian group $G$ divisible by 2.

Proof. Enlarge $M^{4}$ to $N^{4} \sim M^{4}$. Let $A_{1}$ be the 2-skeleton of $S^{4}-$ int $N^{4}$. One can define inductively a sequence of 2-polyhedra $A_{i}$ and their regular neighborhoods $X_{i}$ in $S^{4}-$ int $N^{4}$ as follows:

1. Choose a triangulation $L_{i}$ of $X_{i}$ of mesh at most $1 / i$.

2. Given a 2-simplex $\Delta \subset A_{i}$ of $L_{i}$, find a real projective plane $P(\Delta)$ contained in a 4-simplex $\Delta^{\prime}$ of $L_{i}$ so that $\partial \Delta$ represents the generator of the fundamental group of $P(\Delta)$ and $P(\Delta)-\partial \Delta \subset$ int $\Delta^{\prime}$. We may assume that

Received by the editors August 21, 1992.

1991 Mathematics Subject Classification. Primary 54F45, 55M10.

Key words and phrases. Dimension, cohomological dimension, Union Theorem.

Supported in part by a grant from the National Science Foundation. 
$\left(P\left(\Delta_{1}\right)-\partial \Delta_{1}\right) \cap\left(P\left(\Delta_{2}\right)-\partial \Delta_{2}\right)=\varnothing$ if $\Delta_{1} \neq \Delta_{2}$ (strictly speaking, all we need is that $\left(P\left(\Delta_{1}\right)-\partial \Delta_{1}\right) \cap\left(P\left(\Delta_{2}\right)-\partial \Delta_{2}\right)$ is at most 0-dimensional).

3. Obtain $A_{i+1}$ from $A_{i}$ by removing the interior of $\Delta$ and adding $P(\Delta)$ for each 2-simplex of $A_{i}$.

4. $X_{i+1}$ is a regular neighborhood of $A_{i+1}$ in $X_{i}$ and is contained in the (1/i)-neighborhood of $A_{i+1}$.

Notice that the inclusion-induced homomorphism

$$
H_{2}\left(X_{i+1} ; G\right) \rightarrow H_{2}\left(X_{i} ; G\right)
$$

is an epimorphism for any group $G$ divisible by 2 . Indeed, if $\sum c_{i} \Delta_{i}$ is a 2-cycle in $A_{i}$, we find $d_{i} \in G$ with $2 d_{i}=c_{i}$. Since $c_{i} \Delta_{i}-d_{i} m_{i}$ (here $m_{i}$ represents the chain in the projective plane $P\left(\Delta_{i}\right)$ obtained by adding all its 2-simplices so that $\left.\partial m_{i}=2 \partial \Delta_{i}\right)$ is a 2-cycle in a contractible set $\Delta_{i}^{\prime} \subset X_{i}$, it is a boundary in $X_{i}$ and $\sum c_{i} \Delta_{i}$ is homologous to $\sum d_{i} m_{i}$.

Define $X$ as the intersection $\bigcap X_{i}$. Suppose $\alpha \in H^{1}(M ; G)=H^{2}\left(S^{4}, M\right)$. By Lefschetz Duality (see [Sp, p. 297])

$$
H^{2}\left(S^{4}, M\right) \approx H_{2}\left(S^{4}-M ; G\right),
$$

so $\alpha$ is represented by an element $\beta$ of $H_{2}\left(S^{4}-M ; G\right)$. Now, inductively, find $\beta_{i} \in H_{2}\left(X_{i} ; G\right)$ so that $\beta=\beta_{1}$ in $H_{2}\left(S^{4}-M ; G\right)$ and $\beta_{i+1}=\beta_{i}$ in $H_{2}\left(X_{i} ; G\right)$. Dually, one gets elements $\alpha_{i} \in H^{1}\left(S^{4}-X_{i} ; G\right)$ which pasted together form an extension of $\alpha$ to $S^{4}-X$.

Suppose $C$ is a closed subset of $X$ and $\alpha: C \rightarrow R P^{\infty}$ represents an element of $H^{1}(C ; Z / 2)$. There is an $i$ and an extension $\tilde{\alpha}: Y_{i} \rightarrow R P^{\infty}$ of $\alpha$ to a subcomplex $Y_{i}$ of $X_{i}$. We may assume that $Y_{i}$ contains the 1-skeleton of $X_{i}$. Given a 2-simplex $\Delta$ of $A_{i}$ one can extend $\tilde{\alpha} \mid \partial \Delta$ over $P(\Delta)$. Thus, $\alpha$ is extendible over $Y_{i} \cup A_{i+1}$. Extend $\alpha$ over a regular neighborhood of $Y_{i} \cup A_{i+1}$ containing $X_{i+1}$. Thus, $\alpha$ extends over $X$ which proves that $\operatorname{dim}_{\mathbf{Z} / 2} X \leq$ 1 .

Theorem. There is a subset $A$ of $S^{4}$ such that $\operatorname{dim}_{\mathrm{Z} / 2} A=1$ and $\operatorname{dim}_{G}\left(S^{4}-A\right)=1$ for any abelian group $G$ divisible by 2 .

Proof. Choose a countable family $\left\{M_{i}\right\}_{i \geq 1}$ of compact submanifolds of $S^{4}$ so that for any compact subset $C$ of $S^{4}$ and any open set $U$ containing $C$ there is $i$ with $C \subset M_{i} \subset U$. Choose $X_{i} \subset S^{4}-M_{i}$ such that $\operatorname{dim}_{\mathrm{Z} / 2} X_{i} \leq 1$ and

$$
H^{1}\left(S^{4}-X_{i} ; G\right) \rightarrow H^{1}\left(M_{i} ; G\right)
$$

is an epimorphism for any abelian group divisible by 2 . Let $A \supset \bigcup X_{i}$ be a $G_{\delta}$-set so that $\operatorname{dim}_{\mathrm{Z} / 2} A \leq 1$. Given a compact set $C$ in $S^{4}-A$ and $\alpha: C \rightarrow$ $K(G, 1)$ one can find $M_{i}$ containing $C$ and an extension $\tilde{\alpha}: M_{i} \rightarrow K(G, 1)$ of $\alpha$. Since $\tilde{\alpha}$ extends over $S^{4}-X_{i} \supset S^{4}-A$, the proof of $\operatorname{dim}_{G}\left(S^{4}-A\right) \leq 1$ is completed.

\section{REFERENCES}

[DRS] A. Dranishnikov, D. Repovš, and E. Ščepin, On the failure of the Urysohn-Menger sum formula for cohomological dimension, preprint.

[DW] J. Dydak and J. J. Walsh, Aspects of cohomological dimension for principal ideal domains (in preparation). 
[Ku] W. I. Kuzminov, Homological dimension theory, Russian Math. Surveys 23 (1968), 1-45.

[Ru] L. R. Rubin, Characterizing cohomological dimension: The cohomological dimension of $A \cup B$, Topology Appl. 40 (1991), 233-263.

[Sp] E. Spanier, Algebraic topology, McGraw-Hill, New York, 1966.

Department of Mathematics, University of Tennessee, Knoxville, Tennessee 37996

E-mail address: dydak@math.utk.edu 